\title{
Anesthesia techniques for Carpal Tunnel Syndrome Surgery in the Netherlands
}

\section{E. Kropman, MD ${ }^{1,3}$, J. J. Van Wingerden², MD, PhD, E. J. Buijs ${ }^{3}$, MD \\ ${ }^{1}$ University Medical Center, Utrecht, Department of Anesthesiology ${ }^{2}$ Gelre Hospital, Apeldoorn, Department of Plastic Surgery Gelre Hospital, Apeldoorn, Department of Anesthesiology}

\section{Background and Goal}

- Carpal tunnel syndrome (CTS) surgery can be performed with or without an anesthesiologist attending.

- If an anesthesiologist is present, there are various approaches to provide anesthesia for this procedure.

- This study explores current anesthetic practice for CTS surgery.

\section{Materials and Methods}

- Electronic survey questionnaire, distributed in electronic newsletters of two Dutch anesthesiology associations.

- Questions regarding frequency of anesthesia for CTS surgery, and technical aspects of anesthesia techniques.

\section{Results}

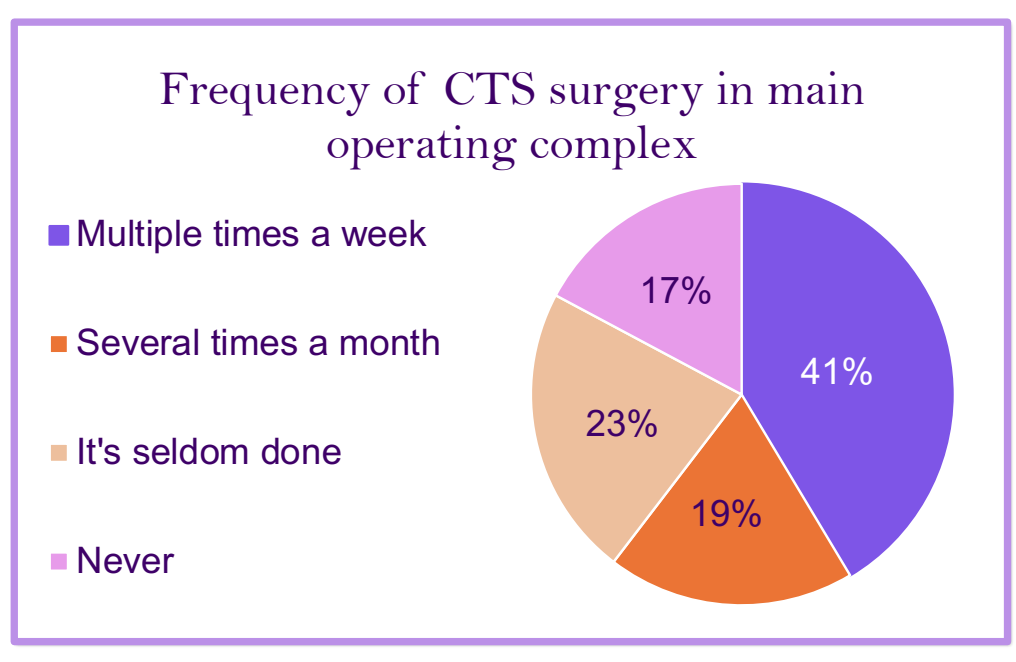

Anesthesia Technique for CTS - Surgeon provides the anesthesia (BPB or local infiltration) Brachial plexus block without sedation

Brachial plexus block with sedation

$\square$ General anesthesia

- Bier block

- Local infiltration anesthesia

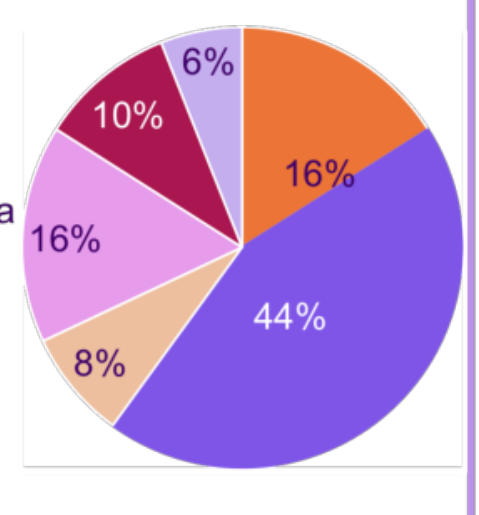

Clinical aids used for blocking brachial plexus in CTS surgery

- Ultrasound only

Ultrasound \& neurostimulator ఐ Neurostimulator only
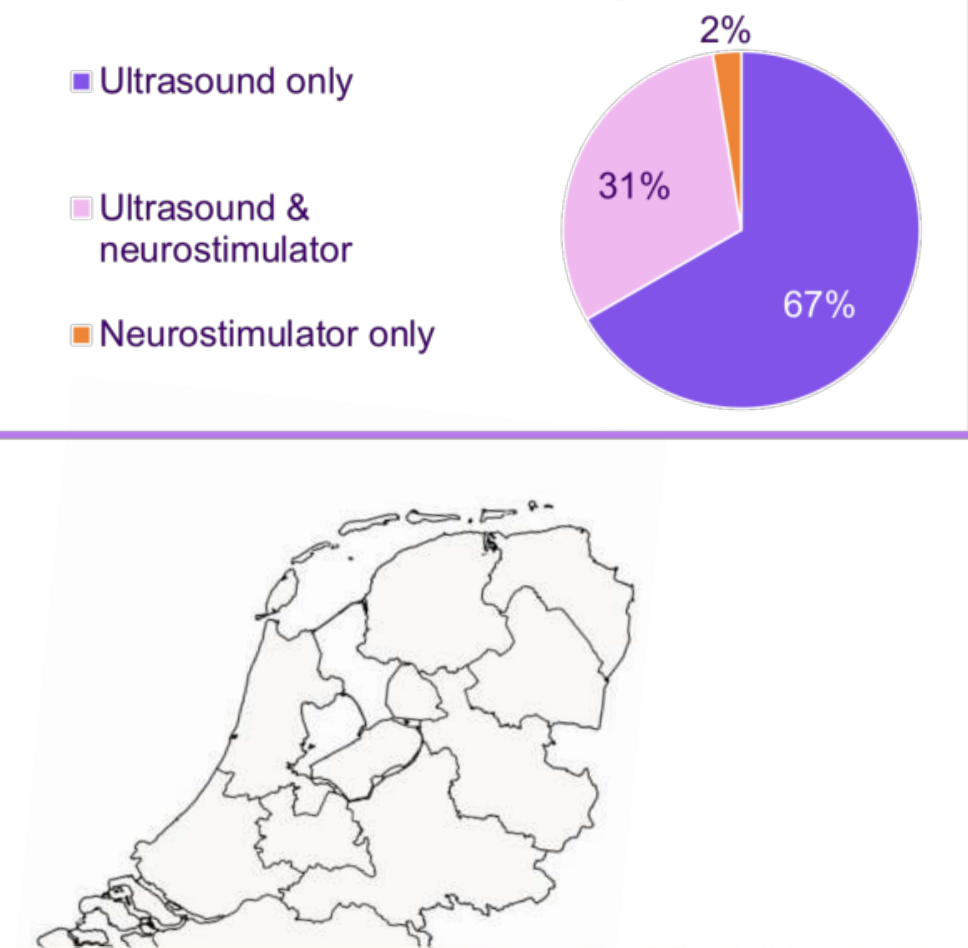

- Responses from 58 anesthesiologists working in 38 different clinics

- Representative of approximately $43 \%$ of official Dutch hospital organizations.

- Carpal tunnel surgery regularly is done in the main operating complex in $60.3 \%$ of clinics.

- Anesthesiologist is involved in $84 \%$ of cases

- $76 \%$ of our respondents used $25 \mathrm{ml}$ or less of local anesthetics, with mepivacaine $1,5 \%$ and ropivacaine $0,75 \%$ being the most popular.

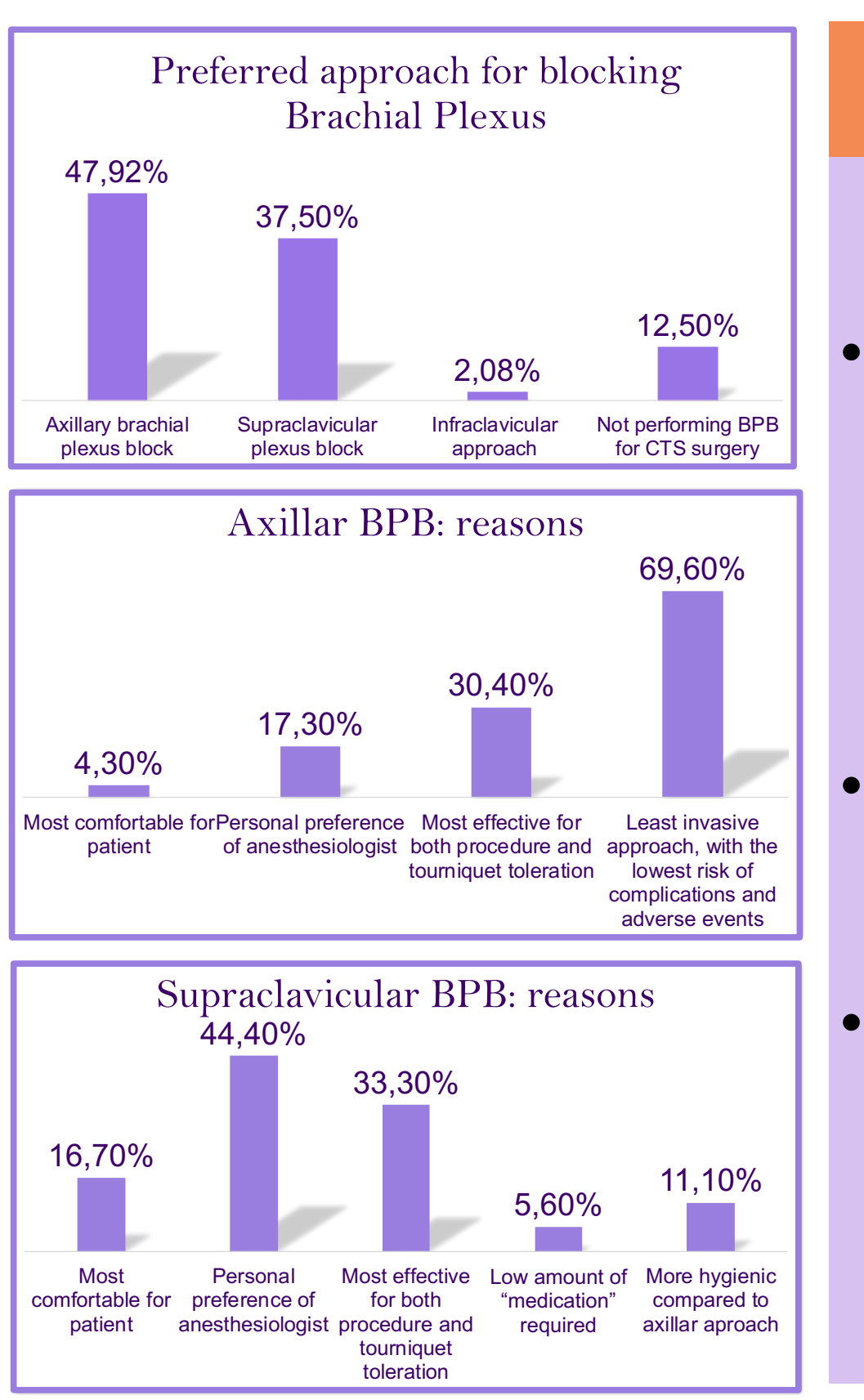

\section{Conclusions}

- In more than half of the clinics studied, carpal tunnel surgery is done in the main operating room with an anesthetist attending.

- $\quad$ The BPB is the most performed anesthesia technique.

- This study could be useful in the development of future guidelines. 